\title{
How do Magnetotactic Bacteria Synthesize Magnetite? - a Soft X-ray Spectroscopy, Spectromicroscopy and Magnetism Time Course Study.
}

\author{
Lucas Le Nagard, ${ }^{1}$ Xiaohui Zhu, ${ }^{1}$ Adam P. Hitchcock, ${ }^{1}$ Dennis A. Bazylinski, ${ }^{2}$ Sufal Swaraj, ${ }^{3}$ Stefan \\ Stanescu $^{3}$ and Rachid Belkhou ${ }^{3}$ \\ 1. Dept. Chemistry \& Chem. Biology, McMaster University, Hamilton, Canada \\ 2. School of Life Sciences, University of Nevada-Las Vegas, Las Vegas, USA \\ 3. Synchrotron Soleil, St. Aubin, France \\ * Corresponding author, aph@mcmaster.ca
}

Prokaryote and archaeon magnetotactic bacteria (MTB) biomineralize chains of single-domain, $\mathrm{Fe}_{3} \mathrm{O}_{4}$ nanoparticles, known as magnetosomes. Magnetosomes, which are arranged in chains inside the cell, function as a strong magnetic dipole so the cell aligns with the earth's magnetic field. Coupled with oxygen sensing (aerotaxis), magneto-aerotaxis is believed to increase the efficiency of the MTB in finding their optimal $\mathrm{O}_{2}$ concentration in the sediments (the interface of oxic and anoxic zones) [1]. As one of the simplest biomineralizing microorganisms, MTB are an ideal model to study biomineralization mechanisms. Although the genetic control is actively under study and a conserved core of genes has been identified, the chemical steps in magnetosome biomineralization are still not fully understood. Several competing hypotheses exist. Frankel et al. [2] reported that $\left(\mathrm{Fe}^{3+}\right)_{2} \mathrm{O}_{3} \cdot 0.5 \mathrm{H}_{2} \mathrm{O}$, an amorphous ferrihydrite, precursor, is formed first, which is then partially reduced and dehydrated to $\mathrm{Fe}_{3} \mathrm{O}_{4}$. However, Faivre et al. [3] did not observe any mineral precursor in a time-resolved Mössbauer study and suggested that $\mathrm{Fe}(\mathrm{II})$ and $\mathrm{Fe}(\mathrm{III})$ quickly co-precipitate to directly form $\mathrm{Fe}_{3} \mathrm{O}_{4}$ within magnetosome vesicles. In a recent study [4], we observed hematite $\left(\alpha-\mathrm{Fe}_{2} \mathrm{O}_{3}\right)$ in MV-1 MTB, which was likely a precursor in magnetosome $\mathrm{Fe}_{3} \mathrm{O}_{4}$ formation. We are investigating biomineralization in AMB-1 MTB cells using Fe L-edge X-ray absorption spectra (XAS) and X-ray magnetic circular dichroism (XMCD) of individual cells and magnetosomes measured by Scanning Transmission X-ray Microscopy (STXM). The measurements are made at the Hermes STXM at Soleil, as well as at CLS aSTXM. We use a timecourse approach. We start with non-magnetic cells (grown in absence of Fe); initiate magnetosome formation by adding $\mathrm{Fe}$ to the culture; and sample at various stages of magnetosome growth and chain formation. Once dried on a TEM grid the cells are dead and stable. By measuring the Fe chemistry inside representative cells, and the magnetism of nascent magnetosomes, we probe how MTB synthesize $\mathrm{Fe}_{3} \mathrm{O}_{4}$ magnetosomes. A combined TEM, XAS study of AMB-1 time course samples was reported earlier [5], but they only measured average Fe L-spectra of many cells, not inside individual cells.

We sampled the time course at 0 min (just before adding the Fe-depleted cells to Fe-containing media), $10 \mathrm{~min}, 30 \mathrm{~min}, 1 \mathrm{hr}, 2 \mathrm{hr}, 4 \mathrm{hr}, 8 \mathrm{hr}$ and $48 \mathrm{hr}$. Fe L2,3-edge XAS measurements were performed on these AMB-1 samples. Transmission electron microscopy of the time course samples at McMaster showed that magnetosomes appeared in some cells after 4-8 hours. Fe L $_{3}$ STXM-XAS results (single spectra at $709.2 \mathrm{eV}$, the $\mathrm{L}_{3}$ peak; Fe maps from 2 images $\left(\mathrm{OD}_{709}-\mathrm{OD}_{704}\right)$; or full spectra fit) at Soleil showed that near complete magnetosomes were present at $4 \mathrm{hr}$, although many of them were very small and had a different spectrum from magnetite. Only a few immature, isolated magnetosomes were observed in the 1 or $2 \mathrm{hr}$ samples. By $48 \mathrm{hr}$, the cells examined were indistinguishable from normally cultured AMB-1 cells, both in terms of imaging (Fig. 1), and Fe $\mathrm{L}_{3}$ spectra (Fig. 2). The 8 hr sample showed immature magnetosomes in a disorganized pattern as well as short chains of normal magnetite. A weak cytoplasmic signal, with clear Fe(III) character, was observed in the $2 \mathrm{hr}$ and $4 \mathrm{hr}$ samples. 
A challenge of our first set of measurements was the presence of culture salts in the samples. Since then we have greatly improved sample preparation by careful washing and by fixation (Fig. 3). Although results to date deal mostly with method development and anecdotal evidence, it is already clear that the time-course method applied to individual AMB-1 cells will be an effective tool to systematically probe how magnetotactic bacteria take up $\mathrm{Fe}$ ions from the local environment, and transform them into $\mathrm{Fe}_{3} \mathrm{O}_{4}$ magnetosomes. Results are expected to address the following questions. Are there precursor cytoplasmic solution or solid species formed before mature magnetosome formation? If so, what is the chemical state of these precursors? How, and at what rate, are these precursors converted into mature magnetite magnetosomes? [6]

\section{References:}

[1] D. Faivre and D. Schuler, Chem.Rev.108 (2008) 4875

[2] R. B. Frankel, et al., Biochim. Biophys. Acta 763 (1983) 147

[3] D. Faivre, et al., Angew. Chem., Int. Ed. Engl 46 (2007) 8495

[4] X.H. Zhu, et al. , Proc. Nat. Acad. Sci 113 (2016) E8219

[5] S. Stanisland, et al., Proc. Nat. Acad. Sci. 104 (2007) 19524

[6] CLS is supported by CFI. We thank Soleil for beamtime.
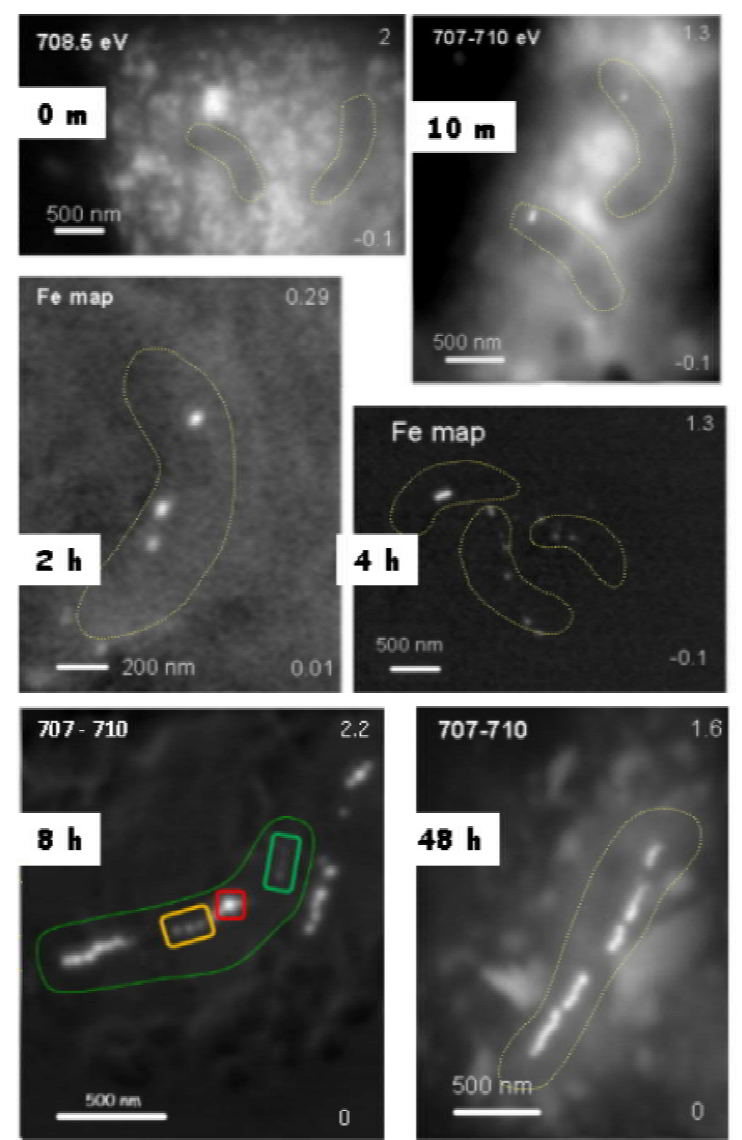

Fig. 1. Single or multi energy images $(0,10 \mathrm{~m} ; 8 \mathrm{hr}, 48 \mathrm{hr})$ or Fe maps from fits $(2 \mathrm{hr}, 4 \mathrm{hr})$ of time course AMB-1 cells derived from $\mathrm{Fe}$ L3 stacks, Cell outlines derived from TEM images of same area. 3 colored areas in 1 cell of the $8 \mathrm{hr}$ sample have different $\mathrm{Fe} \mathrm{L}_{3}$ spectra. (Soleil)

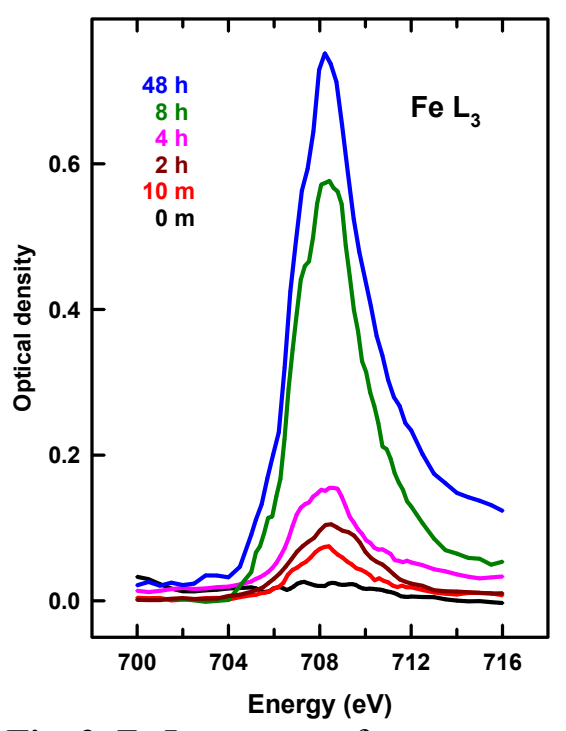

Fig. 2. Fe L3 spectra of magnetosomes or precursors (Soleil).

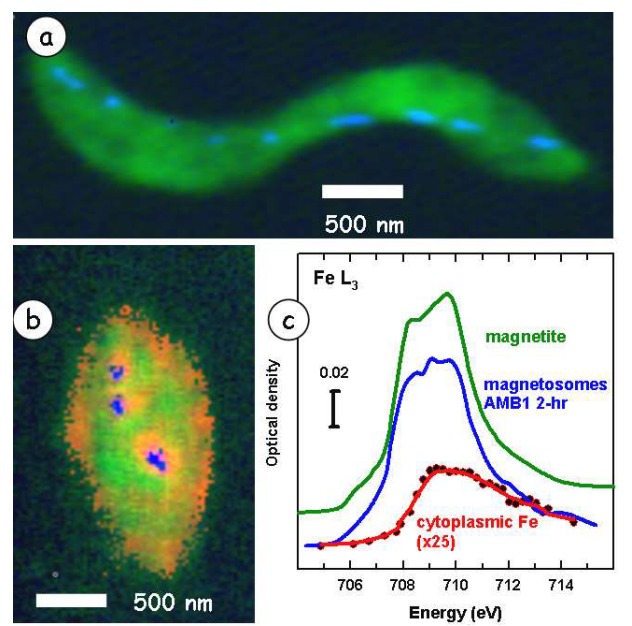

Fig. 3. Fe L3 analysis of improved AMB-1 cell preparation (no extra-cellular materials). (a) gluteraldehyde fixed 48-hr time course (green cell, blue - mature magnetosomes) . (b) phosphate buffer wash, 2-hr time course (green cell, red-cyto-Fe, blue - early magnetosomes). (c) Comparison of $\mathrm{Fe} \mathrm{L}_{3}$ spectra of pure magnetite (blue), magnetosomes in 2-hr sample (green), and cytoplasmic Fe (red). (CLS) 